\title{
LAS FORMAS DE SEGUNDA PERSONA SINGULAR COMO ESTRATEGIAS EVIDENCIALES*
}

\author{
THE FORMS OF SECOND SINGULAR PERSON AS \\ EVIDENTIAL STRATEGIES
}

EVELYN HUGO ROJAS

Pontificia Universidad Católica de Chile. Santiago, Chile

eshugo@uc.cl

\section{RESUMEN}

En esta investigación se estudian las formas de segunda persona singular que no corresponden al dominio de la deixis y que presentan el rasgo de "generalidad" y "encubrimiento del emisor". Se explican estos dos fenómenos en el marco de la evidencialidad a través de los ejes "fuente de información" y "acceso a la información", descritos por Bermúdez (2005). Junto con una propuesta del posible significado de esta forma en el marco evidencial, se realiza un estudio de los aspectos sintácticos y morfológicos que influyen en su interpretación, en una muestra de un corpus del español de Chile, Codicach (Corpus dinámico del castellano de Chile). Se llega a la conclusión de que los elementos más influyentes para la interpretación genérica (acceso compartido) son los activadores genéricos y que el aspecto imperfectivo y el tiempo presente no son requisitos indispensables para que se produzca esta interpretación.

Palabras clave: Evidencialidad, estrategias evidenciales, segunda persona singular no deíctica.

\section{ABSTRACT}

This investigation studies the forms of second singular person which do not correspond to deixis, and present a mark of "generality" and "concealed emitter". These two phenomena are explained according to evidence through the axes of "information source" and "information access", described by Bermúdez (2005). Along with a proposal for the possible meaning of these forms in the evidential context, a study is carried out on the syntactic and morphologic aspects which influence its interpretation in a sample from a Chilean Spanish corpus, Codicach (Corpus dinámico del castellano de Chile). The

* Esta investigación está enmarcada en el proyecto Fondecyt 11070002 "Formas gramaticales de expresión de la evidencia en el español de Chile". 
conclusion is that the most influential elements for the generic interpretation (shared access) are the generic activators and that the imperfective aspect and present tense are not indispensable requirements to reach such interpretation.

Keywords: Evidentiality, evidential strategies, non deictic singular second person.

Recibido: 20/07/2010. Aceptado: 10/01/2011

\section{INTRODUCCIÓN}

T as personas gramaticales y los pronombres personales han sido descritos como שelementos deícticos. Es decir, como unidades cuyo significado se actualiza en cada acto comunicativo a través de la relación con un hablante ( $1^{\mathrm{a}}$ persona singular), con un oyente ( $2^{\text {a }}$ persona singular) o con algo que no es ni lo primero, ni lo segundo ( $3^{a}$ persona singular). Este uso deíctico se ejemplifica a través de un enunciado del corpus Codicach (Corpus dinámico del castellano de Chile) ${ }^{1}$ :

\section{(1) Hola Camila cómo estás? Harto tiempo? Te hiciste humo?}

En este caso, los morfemas de $2^{\text {a }}$ persona singular, presentes en los verbos y en el pronombre átono "te", hacen referencia a un oyente que en la situación comunicativa es "Camila". Este es uno de los enunciados que la tradición gramatical ha determinado como un uso deíctico. El ejemplo (1), sin embargo, parece ser muy distinto del (2), también extraído del mismo corpus:

(2) No son nadie para intentar imponer a la gente su forma de pensar. Talvez es gente que no tiene mayor educación. Con esto uno no puede salir tranquilo, por temor a que estos grupos te ataquen.

En (2) la forma "te" no parece hacer referencia a un oyente. Por lo tanto, no sería un deíctico de persona, ya que quien puede ser atacado no es solo el oyente, sino el hablante y todos quienes compartan aquella experiencia. ¿Qué significado transmite esta forma de $2^{\text {a }}$ persona singular? En este artículo se plantea que casos como (2) pueden ser descritos en el marco del dominio semántico de la evidencialidad.

De esta manera, el objetivo de la investigación realizada fue determinar si las formas de $2^{\text {a }}$ persona singular pueden transmitir un significado evidencial. En caso afirmativo, precisar de qué tipo de significado se trataba en relación con los ejes "fuente de información" y "acceso a la información” y, así, contribuir a la

${ }^{1}$ Se mantendrá la ortografía y tipografía original en todos los casos presentados de este corpus. 
descripción del dominio semántico de la evidencialidad en el español de Chile.

Para lograr este objetivo se analizaron las formas de $2^{\text {a }}$ persona singular no deícticas en una muestra del Codicach y se describieron en términos de sus entornos temporales y aspectuales. Asimismo, se estudiaron las relaciones de esta forma con expresiones tendientes a activar la interpretación evidencial y la coaparición con otras formas evidenciales o de significado genérico.

Por otra parte se analizaron las diferencias generadas en un enunciado por la ausencia o presencia del pronombre personal de $2^{\mathrm{a}}$ persona con interpretación evidencial.

Finalmente, se analizó si la función de las formas de $2^{\text {a }}$ persona singular influye en la interpretación evidencial.

\section{MARCO TEÓRICO}

\subsection{La evidencialidad}

En el lenguaje cotidiano, y en el legal, el término evidencia se asocia con un objeto, dato o argumento que puede ser utilizado para probar la ocurrencia de un suceso. En la lingüística, el significado de "evidencia" y "evidencialidad" difiere en forma drástica: "linguistic evidentiality has nothing to do with providing proof in court or in argument, or indicating what is true and what is not, or indicating one's belief. All evidentiality does is supply the information source. The ways in which information is acquired -by seeing, hearing, or in any another way- is its core meaning" (Aikhenvald, 2004: 4).

Desde la perspectiva de Aikhenvald (2004), al dominio semántico cuyo significado esencial es la indicación de la fuente de la información se le llama "evidencialidad" y se utiliza el término "evidencial" para designar al morfema que expresa dicho significado.

El fenómeno en cuestión ha sido estudiado desde el siglo XVI. Entre sus primeras consignaciones se puede mencionar la realizada por Domingo Santo Thomas en la Gramática o arte de la lengua general de los indios de los reynos del Perú. En ella agrupa a las partículas evidenciales junto con otros morfemas que no se adecúan a la descripción o al modelo de las lenguas romances; las considera como partículas que "de suyo nada significan: pero adornan, o ayudan a la significación de los nombres, o verbos que se añaden" (1951: 14). La concepción de estos morfemas como partículas que no significan nada, señala Aikhenvald, continuó hasta que Boas las describió como evidenciales, momento en que pasan de ser consideradas ornamentales a una "rareza» exclusiva de algunas cuantas lenguas: "while for us definitiness, number, and time, are obligatory aspects, we find in another language location near the speaker or somewhere else, [and] source of information 
-whether seen, heard, or inferred- as obligatory aspects" (1938: 133).

Como se mencionó, sólo en algunas lenguas los hablantes se ven obligados a entregar datos sobre la fuente de la información expresada en su enunciado. Aikhenvald indica que estas lenguas corresponden aproximadamente a un cuarto de las existentes en el mundo: "in about a quarter of the world's languages every statement must specify the type of source on which it is based, for example, whether the speaker saw it, or heard it, or inferred it from indirect evidence, or learnt it from someone else" (2004: 1). Este 25\% corresponde en su mayoría a lenguas "exóticas", como las de la familia tibeto-burmana o lenguas amerindias como el tagalo o el quechua.

Esto último genera la idea de que el dominio evidencial no está presente en lenguas como el español, el francés, el inglés o en las que no se expresa por medio de morfemas obligatorios: "gramatical evidentiality is not a terribly frequent phenomenom. (...) Most familiar languages -such as English, or French- lack it" (Aikhenvald, 2004: 17). Para Bermúdez (2005), la propuesta de Aikhenvald llevaría a pensar que si una lengua marca gramaticalmente la fuente de información opcionalmente, o si la marcación evidencial se realiza mediante dispositivos gramaticales no morfológicos o mediante partículas evidenciales que no forman sistema, ésta posee sólo "estrategias de evidencialidad", o que tiene "otras categorías gramaticales que pueden adquirir significados evidencialoides como 'efecto colateral', sin tener la 'fuente de información' como su significado primario.

Bermúdez (2005) entrega un argumento que cuestiona este planteamiento que lleva a realizar una división entre lenguas evidenciales y no evidenciales. Él señala que la naturaleza gramatical de una forma lingüística es siempre una cuestión de grado, por lo tanto no sería posible trazar una clara línea divisoria entre gramática y léxico. De esta manera, muchas de las formas lingüísticas que se consideran léxicas son, en mayor o menor grado, gramaticales, y viceversa.

Quienes apoyan la postura diferenciadora argumentan que si se aceptan como evidenciales los fenómenos que, aunque relacionados con la indicación de la fuente de información no son morfológicos, se corre el riesgo de viciar la descripción del dominio evidencial. Bermúdez contraargumenta señalando que esto sería equivalente a decir que la investigación de la modalidad como dominio semántico y su estudio en uso (adverbios, conectores, marcadores discursivos, entre otros) oscurecería el estudio del modo verbal. Concluye que los estudios sobre evidencialidad morfológicamente codificada y evidencialidad codificada de otras formas gramaticales o de manera opcional pueden coexistir y son complementarios, del mismo modo que los estudios sobre los verbos modales, sobre el modo verbal y sobre la modalidad coexisten y se complementan.

De acuerdo con lo anterior, los marcadores evidenciales (gramaticales o léxicos) pueden ser definidos como "formas lingüísticas cuyo significado es una referencia acerca de la fuente de información de la proposición” (Bybee, 1985 en Bermúdez, 
2005: 5). Las posibilidades, indica Bermúdez, son varias: haber tenido contacto directo (visual o de otro tipo) con la situación descrita; haber tenido contacto no con la situación misma pero sí con indicios que apuntan hacia esa situación o haber recibido información de una tercera persona. En otras palabras, existe la posibilidad de que la información se haya obtenido en forma directa o indirecta.

Bermúdez (2005) considera que además de la fuente de la información (que él codifica como variable entre los polos "ajeno" y "personal") existirían otros dos ámbitos relacionados con el dominio evidencial: el modo de acceso y el acceso a la información. La propuesta esquematizada ${ }^{2}$ se presenta a continuación en la Figura 1:

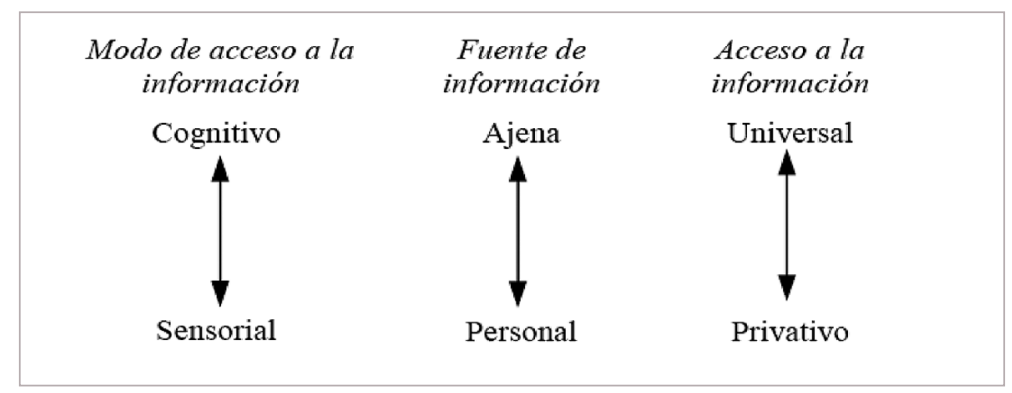

Figura 1: Dominio de la evidencialidad revisado por Bermúdez (2005).

Esta propuesta concuerda con la visión de la lingüística cognitiva respecto de la conformación de categorías, lo que permite -explica Bermúdez- verlas como entidades de límites difusos con zonas centrales, periferia y zonas de transición y no como conjuntos cerrados de miembros definidos claramente por condiciones necesarias y suficientes (Rosch 1975; Rosch \& Mervis, 1978; Lakoff, 1987 en Bermúdez, 2005), lo que parece acercarse mucho más a la forma de aprehender el mundo que tienen las personas. Desde esta perspectiva, "una categoría posee elementos o miembros más característicos, llamados prototipos, y miembros más

${ }^{2}$ Bermúdez en esta propuesta considera las clasificaciones de Willet (1998), quien subdivide la evidencialidad en directa e indirecta y la de Plugian (2001), quien indica que existen lenguas que oponen evidencia directa a indirecta; otras que consideran tres tipos: evidencia directa, razonada y mediada; y otras que la clasifican en evidencia personal y mediada. Bermúdez señala que estas clasificaciones, y algunas otras (Aikhenvald 2003, 2004; Anderson, 1986) son restringidas y rígidas, al dejar fuera aspectos relacionados con el acceso a la información y al no captar el carácter continuo de las magnitudes que conforman la evidencialidad. Ante esto, propone un esquema de ejes continuos y también incluye un tercer aspecto denominado "acceso a la información" que contempla lo que algunas clasificaciones (Willet, 1998; Lazard, 2001) consideran como "folklore" y que se situarían en el polo universal del continuo entre universal y privativo. 
periféricos, que suelen constituir un paso gradual hacia otra categoría, que a su vez posee su propio centro o foco" (Bermúdez, 2005: 11).

Si se vuelve a la Figura 1, se aprecia que existe un eje denominado "modo de acceso a la información" que corresponde al continuo entre lo sensorial y lo cognitivo. Este respondería a la pregunta ¿cómo se obtuvo la información? Junto a este eje se encontraría el correspondiente a la "fuente de la información", la que es conceptualizada como un continuo entre la información personal y la ajena (el dónde de la información). Por último, se puede observar la propuesta de un tercer eje denominado "acceso a la información", que contempla las opciones que van desde el acceso privativo al acceso universal a la información (¿quiénes?).

Se considera que esta propuesta describe en forma adecuada el dominio de la evidencialidad, por lo tanto, será tomada como base para la descripción del fenómeno estudiado en esta investigación.

\subsection{La evidencialidad en el español}

Como se señaló anteriormente, el español no es una lengua que deba indicar de forma obligatoria datos acerca de la fuente de la información. Sin embargo, sí existen estrategias sistemáticas para expresar la evidencialidad. Entre los casos que han sido estudiados como estrategias evidenciales destacan: algunos tiempos verbales (el condicional, el futuro, el pretérito imperfecto), construcción verbo de percepción + cláusula subordinada y algunos conectores. A continuación son ejemplificados estos fenómenos:

(3) Disco "Pare" no habría sido respetado, según Ferrocarriles.

(4) Está temblando: tendrá frío.

(5) Será socialista, pero su dinero no lo quiere compartir.

(6) El tren salía a las 5.

(7) Oí ganar a los jóvenes.

(8) Me trataron mal de ahí que me fui.

En el ejemplo (3), denominado en la tradición gramatical «condicional de rumor», como explican González y Lima (2009), resulta claro que la forma verbal no expresa significados temporales (antepospretérito, en este caso), "ni tampoco aparece en la apódosis de una estructura condicional, sino que su valor es el de señalar que lo que se comunica es información de segunda mano; (...) incluso, esta fuente externa es señalada explícitamente: «Ferrocarriles»" (2009: 6). Es decir, el caso del condicional sería relevante a partir del eje «fuente de la información».

En (4) el significado parece ser el de inferencia: "al usar el futuro, el hablante indica que la información no proviene de la experiencia directa sensorial sino de 
un proceso inferencial generado a partir de indicios [tiembla]" (Bermúdez, 2005: 144). En el caso de (5) el hablante problematiza un hecho, poniéndolo en duda. Nuevamente el eje "fuente de información» se torna relevante en la interpretación: la fuente es ajena.

En el caso (6) se aprecia un morfema de pretérito imperfecto que no indica un evento pasado: el hablante podría emitir (6) si, por ejemplo, acaba de leer la información en un horario de salidas, y seguidamente se lo comunica a alguien. Al usar el imperfecto el hablante está comunicando aquí que la información es indirecta.

(7) es un caso de construcción con verbos de percepción y cláusula subordinada. La información sólo pudo haberse adquirido de fuente directa. Esto se deduce por contraste con "oí que los jóvenes ganaron"; en esta oración puede interpretarse que el hablante recibe directamente la información a través de los sentidos o que alguien le transmite ese conocimiento. En el ejemplo (7) esta última interpretación no es posible, con la consecuente relevancia del eje «modo de acceso a la información» en el polo de lo sensorial.

Por último, en el caso (8) se presenta un conector consecutivo "de ahí que», que al observarlo en contraste con otras unidades de su paradigma, es interpretado como un evidencial. Los siguientes ejemplos son entregados por Bermúdez:

(9) Me trataron mal, asi que me fui.

(10) Me trataron mal, por eso me fui.

En los tres casos el hablante afirma que el hecho de haberse ido fue una consecuencia de la manera en la que fue tratado. Sin embargo, en (9) presenta el hecho como una información nueva para el oyente, mientras en (10) la presenta como una información a la que tanto hablante como oyente tienen acceso. En (8) ambas interpretaciones son posibles. La oposición presentada, entre acceso privativo o acceso compartido a la información, es evidencial en el sentido de que se relaciona con la fuente de la información y el acceso de los participantes a ella: tanto el hablante como el oyente lo tienen en (8) y (10), sólo el hablante en (9) 3 .

Se considera que estos estudios son aproximaciones bastante consistentes al fenómeno de la evidencialidad en el español. Los casos presentados son una demostración de que en esta lengua existen elementos sistemáticos de expresión de la evidencialidad, por lo que se hace necesario realizar investigaciones que afinquen estas primeras descripciones y que aborden la tarea de descubrir nuevas estrategias evidenciales.

${ }^{3} \mathrm{Si}$ bien estos fenómenos podrían ser explicados apelando a las nociones de foco y presuposición, al haber una relación con la fuente de la información y el acceso a ella, es posible también analizarlos desde la perspectiva de la evidencialidad. 


\subsection{La evidencialidad en el español de Chile}

En el español de Chile no se encuentran muchos estudios sobre la evidencialidad. Es una excepción la investigación de González y Lima (2009), en la que se describe el uso del pronombre «uno» evidencial, en casos como (11):

(11) uno tiene que sufrir en esta vida para ser feliz después.

Ellos plantean que el uso evidencial del pronombre se observa claramente si se contrasta con otras unidades alternantes en el paradigma, como serían los casos (12a) y (12b):

(12) a. yo tengo que sufrir en esta vida para ser feliz después.

b. el hombre tiene que sufrir en esta vida para ser feliz después.

Estos investigadores proponen que el significado evidencial de la forma «uno» se basa en la interacción entre los ejes 'fuente de información' y 'acceso a la información': "si el hablante elige el pronombre «yo» el significado que transmite es que la fuente de información es 'personal' y que el acceso a la información se considera 'privativo' [esto] indica que la información contenida en el enunciado proviene de la experiencia o creencia personal, la que no se presume necesariamente como accesible para su interlocutor" (p. 12).

Si el hablante escoge una frase nominal [como «el hombre» en (12b)] altera las posiciones del esquema: se vuelve neutral en cuanto a la fuente de información (no indica si es privativa o ajena), pero sí especifica que el acceso a esta información es 'universal'; es decir, el hablante señala que lo expresado en el enunciado no proviene necesariamente de su propia experiencia y que la aseveración corresponde a un hecho conocido en forma amplia. Por último, el escoger el pronombre «uno» implica que la fuente de información es 'personal' (al igual que en la elección de «yo»), pero el acceso a la información se considera 'universal' (como en la elección de «el hombre»); en otras palabras, al elegir «uno» el hablante expresa que lo que afirma proviene de sus propias experiencias o creencias, pero que cualquiera podrá estar de acuerdo con él si atiende a las propias, como se observa en el ejemplo (11). Los investigadores concluyen que al conjugarse el acceso universal y la fuente personal en una sola forma («uno»), ésta presentaría ventajas retóricas.

\subsection{Las formas de $2^{a}$ persona singular en usos no deícticos}

Es interesante observar cuáles han sido las diversas explicaciones que se han dado para explicar los usos no deícticos de las formas de $2^{\text {a }}$ persona singular en distintos momentos de la lingüística. Fernández Ramírez (1986) menciona que el morfema 
de $2^{\text {a }}$ persona singular es otra unidad que junto a «uno» puede adquirir una significación de generalidad:

(13) ¡Y cuidado si es difícil esto de la felicidad de un pueblo! Porque uno viene $\mathrm{y}$ te dice una cosa, y luego entra otro y te dice otra cosa.

En el ejemplo anterior, la forma «te» no parece hacer referencia a un oyente: "pero los pronombres no siempre tienen antecedente (no son deícticos), sino que a veces pueden tener referencia genérica, remitir a todo un grupo más o menos amplio de individuos. El pronombre se interpreta en estos casos como un cuantificador universal similar a cualquiera / todo el mundo" (Fernández Soriano, 1999: 1217).

En la misma línea, Briz, en su libro El español coloquial: situación y uso, dedica un apartado al tema de la deixis personal "concretamente en el uso de los pronombres de $1^{\text {a }}$ y $2^{a}$ persona, las voces principales del diálogo" (1998: 56). Él señala que aunque el centro deíctico personal de la conversación coloquial es el "yo», en ocasiones parece desplazarse: "Así sucede en los casos de impersonalización mediante la forma se, los indefinidos uno, una o el uso del denominado 'tú'" (1998: 56). Como ejemplo de este último propone:

(14) Hay cosas que tú vas aguantando y las vas aguantando un día y dos y tres y cuatro pero llega un día que ya no puedes más y dices...

En este caso se señala que "el yo aparece latente bajo la máscara de la impersonalidad o del propio tú, en un intento de atenuar o minimizar su papel" (Briz, 1998: 57). Así concluye que "como puede notarse, el yo, con una intención persuasiva, presenta de forma confusa la referencia deíctico-personal a la hora de adjudicar a alguien lo que se va a decir: 'no soy yo, sino nosotros', 'somos todos y ninguno', 'es cualquiera', 'eres tú también'. En tales casos, todo parece indicar que el yo intenta salvaguardar su imagen respecto al interlocutor" (1998: 57).

Hernanz menciona que lo que resulta llamativo del empleo de la forma singular para encubrir al emisor es que pueda conjugarse con la interpretación básicamente general que éste posee, es decir que la interpretación de encubrimiento del "yo" y lo genérico no serían excluyentes: "lo que parece existir es una estrategia para 'eludir responsabilidades', al menos en forma directa. Dicha estrategia consiste, fundamentalmente, en encubrir su propia individualidad amparándose en una referencia que le incluya a él y a otros que podrían hallarse en circunstancias semejantes a las suyas" (1990: 163).

Casos como (14) son denominados por Benveniste como una variedad de impersonalidad: "la definición de la $2^{\text {a }}$ persona como aquella a la que la primera se dirige conviene sin duda a su empleo más ordinario. Pero ordinario no quiere 
decir único y constante. Puede usarse la $2^{a}$ persona fuera de la alocución y hacerla entrar en una variedad de "impersonal" (1997: 167).

Existen autores que critican la inclusión de estas formas dentro de las impersonales, entre ellos Vila: "hay que evitar presentarlo en el marco restringido de las construcciones impersonales, porque trasciende al sujeto y a la flexión personal del verbo. Puede quedar afectado cualquiera de los signos con morfema de persona" (1987: 58). Ciertamente el uso no deíctico de la $2^{a}$ persona singular se da en distintas funciones, pero esto no imposibilita la relación del fenómeno con la impersonalidad si se distinguen dos tipos de ésta, como lo hace Barrajón (2005): “...estos casos no responden a una impersonalidad gramatical o sintáctica, [hay] un sujeto léxico que puede estar explícito o implícito y que es el pronombre de segunda persona singular tú (...), su referencialidad se ve diluida hasta el punto de recibir una interpretación indefinida o genérica, con lo que estaríamos ante un caso de impersonalidad semántica" (2005: 47).

Para las formas de $2^{a}$ persona singular, en resumen, se han indicado, desde diversas perspectivas, dos posibilidades de interpretación: la generalidad y el encubrimiento de "yo» (Hernanz, 1990; Hollaender Jensen, 2002 y Barrajón, 2005).

El uso del «tú» encubridor del "yo» se caracterizaría, en opinión de Hollaender Jensen, por ser empático, en tanto que se apela al interlocutor "a que participe de manera más activa en el contenido de lo comunicado (...), que de alguna manera se imagine en las mismas circunstancias" (2002: 130). Para emitir el juicio anterior, el autor se basa en la propuesta de Vila (1987), quien señala que al usar una segunda persona singular no deíctica el emisor intenta hacer menos subjetivo su discurso, utilizando la forma más adecuada para que el oyente se sienta involucrado en su propia forma, la de segunda persona. Si se vincula lo anterior con las ideas propuestas por Bermúdez acerca de los ejes evidenciales, se advierte una clara relación entre el 'uso genérico' y el 'acceso universal' y el 'encubrimiento del yo' y 'fuente de información persona'. El hecho de poder explicar estos dos significados bajo un solo mecanismo interpretativo (la evidencialidad) permite que la descripción sea más simple y, por ende, más adecuada.

\subsection{Las características sintácticas y morfológicas de las formas de $2^{\mathrm{a}}$ persona singular no deícticas}

Otra propuesta acerca de «uno» y las formas de $2^{a}$ persona singular es la de Hernanz (1990), quien denomina a ambos elementos "singulares arbitrarios" porque compartirían ciertas características, como es la interpretación no referencial, con los llamados "plurales arbitrarios" y con los sujetos arbitrarios en general (impersonales) que corresponden, respectivamente, a casos como los siguientes:

(15) Llaman a la puerta. 
(16) Se come bien en este restaurante.

Si bien estas dos formas "arbitrarias" se asemejan en la interpretación no referencial, son diferentes en otros aspectos. Por ejemplo, los singulares arbitrarios siempre pueden incluir al emisor, a diferencia de los plurales arbitrarios, que siempre lo excluyen y además constituyen el único caso en que un sujeto explícito mantiene la interpretación no referencial (18), lo que no ocurre con los plurales como en (17):

(17) Ellos llaman a la puerta.

(18) Si tú dices esto en una reunión la gente se escandaliza.

Una de las cuestiones más interesantes de la propuesta de Hernanz es el estudio de las particularidades sintácticas de los singulares arbitrarios. Esta autora realiza lo anterior con la intención de demostrar que "obedecen a pautas sintácticas regulares, lo que constituye una prueba importante a favor de un tratamiento 'sistemático' -y no meramente pragmático- del fenómeno que nos ocupa” (1990: 160). El hecho de que el comportamiento de estas formas sea sistemático desde el punto de vista sintáctico, permite, además, legitimar su estudio desde el dominio evidencial. Si se vuelve al apartado 2.1., se puede recordar que el hecho de que una lengua no tenga morfemas evidenciales de forma obligatoria y exclusiva no es un impedimento para afirmar que esa lengua posee un dominio evidencial, digno de ser analizado. Sin embargo, esto último es cierto en tanto la estrategia evidencial sea sistemática. De esta manera, el descubrir y describir las regularidades sintácticas de los singulares arbitrarios es un elemento favorable para la adscripción del fenómeno al dominio evidencial.

Hernanz propone que entre las características más interesantes de los singulares arbitrarios destaca el carácter defectivo, entendido como la imposibilidad de una forma para ser usada en todos los modos, tiempos o personas: "las razones de esta defectividad hay que buscarlas en el valor genérico, desprovisto de toda referencia temporal definida, que es propio de estas oraciones. Ello hace que sean incompatibles con entornos temporales y aspectuales de carácter puntual" (1990: 156).

(19) En México disfrutas de un clima envidiable.

(20) En México disfrutaste de un clima envidiable.

En (20), demuestra Hernanz, el pretérito perfecto invalidaría la interpretación arbitraria porque circunscribe el evento a un tiempo específico. No obstante, un tiempo con aspecto imperfectivo es condición necesaria, pero no suficiente para legitimar la interpretación arbitraria: es necesaria la adición de determinados elementos tendentes a "activar" el carácter genérico de un enunciado, lo que tiene 
como consecuencia hacer posible la lectura arbitraria. Hernanz distingue tres tipos de activadores genéricos:

a) los verbos modales «poder» $\mathrm{y}$ «tener». Obsérvese el contraste entre estas cuatro oraciones:

(21) Este champú es tan suave que lo usas para los niños.

(22) Este champú es tan suave que puedes usarlo para los niños.

(23) Los libros que lees para el examen de Anatomía son aburridos.

(24) Los libros que tienes que leer para el examen de Anatomía son aburridos.

En (21) y (23) se tienen verbos en presente, pero sólo en (22) y (24) se activa la lectura genérica gracias a los verbos modales «poder» $\mathrm{y}$ «tener».

b) Una segunda clase de activador es el conector si... entonces, que se materializa en oraciones condicionales, como se observa en (25):

(25) Si comes mucho, engordas.

c) Entre estos activadores de la generalidad se encuentran las construcciones «siempre que», "en situaciones de este tipo» como construcciones adverbiales en posición dislocada. Sólo en (26) se da la lectura arbitraria:

(26) En primavera, te sientes optimista.

(27) Te sientes optimista.

Otra característica es la posibilidad que éstos exhiben de coaparecer libremente con otras formas igualmente indefinidas. Esto se aprecia en (28):

(28) Cuando tienes un desengaño se aprende mucho de la vida.

\section{METODOLOGÍA}

\subsection{Descripción del corpus y decisiones muestrales}

En esta investigación se trabajó con el Codicach. Este es un corpus sincrónico, compuesto aproximadamente por 800.000 millones de palabras, las que están organizadas en 122 subcorpus según distintos géneros funcionales del español de Chile escrito, entre los que se encuentran diarios, cartas a medios, artículos científicos, foros de internet, artículos de revista, etc. 
En la investigación se trabajó con una muestra estratificada o muestra por cuota. Se solicitaron muestras aleatorias de algunos géneros. Se presenta a continuación una tabla que resume los tipos de textos solicitados, la cantidad de archivos (textos) y el número de palabras de éstos.

Tabla I. Corpus de la investigación según número de textos y palabras.

\begin{tabular}{|l|c|c|}
\hline Tipos de textos & $\mathrm{N}^{\circ}$ de textos & $\mathrm{N}^{\circ}$ palabras \\
\hline Cartas a medios (CaM) & 100 & 58.423 \\
\hline Diarios (D) & 100 & 40.256 \\
\hline Foros de internet (FI) & 100 & 86.759 \\
\hline Foros de prensa (FP) & 50 & 84.155 \\
\hline Revistas (R) & 81 & 77.080 \\
\hline Textos académicos (TAP) & 68 & 617.268 \\
\hline Foros Usenet (UN) & 50 & 532.919 \\
\hline Total & 549 & 1.496 .860 \\
\hline
\end{tabular}

\subsection{Procedimiento de identificación y análisis de las formas de $2^{\text {a }}$ persona singular}

Cada manifestación lingüística del morfema de $2^{\text {a }}$ persona singular fue analizada y observada en su contexto con el fin de establecer qué ámbito semántico expresaba. Así, por ejemplo, una forma como «te» fue, en primer lugar, identificada, destacada con mayúsculas y clasificada como correspondiente a una de las posibles formas de expresión del significado evidencial; esta forma fue luego observada en su contexto y se estableció en consecuencia si expresaba un significado deíctico o evidencial.

Es importante aclarar que en esta investigación se dejaron de lado las formas de $2^{\text {a }}$ persona singular posesivas, a pesar de que se encontraron casos que pueden ser considerados evidenciales. Esta opción se tomó para restringir la cantidad de elementos a interpretar y además porque los casos pronominales y las formas verbales son elementos sintáctica y morfológicamente más complejos.

A cada ocurrencia de la forma en cuestión se le asignó un número y se consignó en una tabla, la que facilitó el análisis de los aspectos sintácticos y morfológicos que se describen a continuación. 


\subsection{Análisis de los elementos sintácticos y morfológicos que pueden influir en la interpretación evidencial de la $2^{\text {a }}$ persona singular}

Como se señaló en la introducción, además de precisar qué tipo de significado evidencial expresan las formas de $2^{\text {a }}$ persona singular, interesa describir cuáles son las peculiaridades morfológicas y sintácticas que permitan la interpretación evidencial. Se presentan a continuación las preguntas de investigación que guiaron esta descripción:

a) ¿Qué características tienen los entornos temporales y aspectuales de los casos identificados como estrategias evidenciales?

b) ¿Existen algunas expresiones que tiendan a activar la interpretación evidencial de un enunciado en el que participe una forma de $2^{\text {a }}$ persona singular?

c) ¿La ausencia o presencia del pronombre personal de $2^{a}$ persona en un enunciado influye en la interpretación evidencial del mismo? ¿Esta ausencia o presencia genera matices de significado distinto, aunque ambas sean interpretadas como evidenciales?

d) ¿Las formas de $2^{\text {a }}$ persona singular con interpretación no deíctica coaparecen libremente con otras formas igualmente indefinidas? ¿Esta coaparición influye en la interpretación evidencial del enunciado?

e) Respecto a las formas identificadas como estrategias evidenciales, ¿qué función sintáctica es la predominante? ¿Existe alguna relación entre la función sintáctica de la forma de $2^{\text {a }}$ persona singular y la interpretación de ésta como estrategia evidencial?

A continuación se presenta la tabla utilizada para el análisis de los aspectos sintácticos y morfológicos mencionados en las preguntas anteriores:

Tabla II. Para el análisis de los aspectos sintácticos y morfológicos de las formas evidenciales de $2^{\text {a }}$ persona singular.

\begin{tabular}{|c|c|c|c|c|c|c|c|c|c|}
\hline \multirow{2}{*}{$\mathrm{N}^{\circ}$} & \multicolumn{6}{|c|}{$\begin{array}{l}\text { Forma en contexto: No son nadie para intentar imponer a la gente su forma de pensar. Tal vez es gente que no } \\
\text { tiene mayor educación. Con esto uno no puede salir tranquilo, por temor a que estos grupos TE ataquen. }\end{array}$} \\
\hline \multirow{2}{*}{1} & Dudoso & Forma & Función & $\begin{array}{c}\text { Activador } \\
\text { genérico }\end{array}$ & Coaparición & Tiempo & Aspecto & $\begin{array}{c}\text { Presencia } \\
\text { del p.p. }\end{array}$ & Otros \\
\cline { 2 - 9 } & NO & TE & OD & $\begin{array}{c}\text { poder/ salir } \\
\text { tranquilo }\end{array}$ & uno/ la gente & presente & imperfectivo & n/c & $\mathrm{X}$ \\
\hline
\end{tabular}

En la casilla " $\mathrm{N}^{\circ}$ " se indicó el número que identifica cada caso de $2^{a}$ persona singular interpretado como estrategia evidencial y en "forma en contexto" se presenta éste con su contexto de aparición. En "dudoso" se registra si la interpretación evidencial es dudosa o no. En la casilla "forma" se señala el elemento que en 
la tabla de identificación fue destacado con mayúsculas y en "función" se indica qué función sintáctica desempeña la unidad. En la casilla "activador genérico" se consignan las frases adverbiales, verbos modales (poder y tener) y los conectores del tipo "si... entonces" que pudieran favorecer la interpretación evidencial, si es que los hubiera; si no, se rellena con una X. Por último, en "coaparición" se registra la presencia de otras unidades con significado genérico que podrían influir en la interpretación evidencial de la forma de $2^{\text {a }}$ persona singular (uno, se, frases nominales, etc.).

Si la $2^{\text {a }}$ persona singular es verbal se indica a qué tiempo y aspecto corresponde, y en la casilla "presencia del p. p." se señala si además del verbo conjugado en $2^{\text {a }}$ persona singular aparece un pronombre de $2^{\text {a }}$ persona singular. Si la forma no es verbal se registran las características del entorno de la unidad en cuestión. En este último caso, en la casilla "presencia del p. p.", se pone "n/c", que significa "no corresponde". Finalmente, en la cuadrícula "otros" se agrega alguna información que parezca relevante para la interpretación evidencial.

Posteriormente, a través de la información consignada en esta última tabla se realizó un análisis cuantitativo y cualitativo de las características morfológicas y sintácticas que pueden influir en la interpretación evidencial de las formas de $2^{\text {a }}$ persona singular.

Es importante destacar que el análisis de los aspectos morfológicos y sintácticos se efectuó con posterioridad a la identificación de la forma de $2^{\mathrm{a}}$ persona singular como evidencial. Es decir, primero se identificó como propia de este ámbito y luego se analizó qué factores podrían estar influyendo en esta interpretación. Resultan de especial interés, entonces, las unidades que son clasificadas como dudosas.

\section{RESULTADOS}

En el corpus de la investigación se detectaron 4.988 casos correspondientes a la 2a persona singular, de los cuales 277 poseen una posible interpretación evidencial, lo que equivale al $6 \%$ del total. Esto se consigna en la Tabla III.

Tabla III. Formas de $2^{\text {a }}$ persona singular detectadas en corpus Codicach.

\begin{tabular}{|l|c|c|}
\hline & \multicolumn{1}{|c|}{$\mathrm{N}^{\circ}$} & Porcentaje \\
\hline Deíctico & 4.711 & 94 \\
\hline Evidencial & 277 & 6 \\
\hline Total & 4.988 & 100 \\
\hline
\end{tabular}

De las 277 formas interpretadas como evidenciales, la mayoría se concentra en los textos correspondientes a los usenet $(53 \%)$, seguidos por los foros de internet 
(26\%). Se cree que la causa de estos altos porcentajes se encuentra en la predominancia de la interacción presente en estos géneros. Sin embargo, esta hipótesis debe ser comprobada mediante elementos estadísticos más confiables. En cuanto a los aspectos sintácticos y morfológicos de las formas de $2^{\text {a }}$ persona singular, se observa que la función predominante en el corpus analizado es la de morfema del núcleo de frase verbal, en un $56 \%$ de los casos, seguida por la de objeto indirecto y directo. Lo anterior se aprecia en la Figura 2:

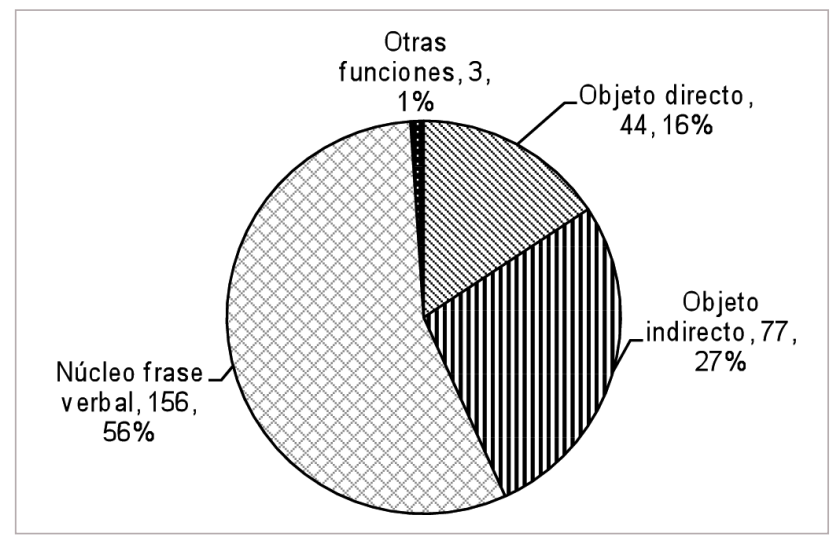

Figura 2. Funciones sintácticas de las formas de segunda persona singular.

Tabla IV. Ejemplos de segunda persona singular con interpretación no deíctica según funciones sintácticas.

\begin{tabular}{|l|c|}
\hline \multicolumn{1}{|c|}{ Ejemplos } & Función \\
\hline $\begin{array}{l}\text { A la primera TE tiran la cadena y listo, por lo tanto claro que me gustaría } \\
\text { que me pagaran algún interés por el saldo diario de mi cuenta corriente. }\end{array}$ & Objeto indirecto \\
\hline $\begin{array}{l}\text { Me quedo con el t28 w, puedes personalizar los ring a la agenda, si TE } \\
\text { llaman de casa suena distinto. tiene el tetris almacena direcciones de correo } \\
\text { etc, hace dos años que me tenia caliente hasta que me lo compre. }\end{array}$ & $\begin{array}{c}\text { Objeto } \\
\text { directo }\end{array}$ \\
\hline $\begin{array}{l}\text { Yo creo que todo pasa por los gustos personales: si te interesa la cosa artistica } \\
\text { dificilmente VAS A ENCONTRARLE algo malo a lo de Tunik o a la Baby }\end{array}$ & $\begin{array}{c}\text { Núcleo frase } \\
\text { verbal }\end{array}$ \\
$\begin{array}{l}\text { Vamp o incluso al gallo de la juguera, dentro de toda la feria de vanidades y } \\
\text { el negociado que se mueve alrededor del arte es siempre muy bueno todo lo } \\
\text { que sale de lo establecido, esa es la fuente de vitalidad del arte tal como lo es } \\
\text { la curiosidad en la ciencia. }\end{array}$ & \\
\hline
\end{tabular}

También se analizó si las formas verbales presentes en el corpus (156 casos) estaban reforzadas por la presencia de un pronombre personal en función sujeto. Esto ocurrió sólo en 17 oportunidades, lo que corresponde a un 10,9\% de los casos verbales y a un $6 \%$ del total de las unidades identificadas como evidenciales. 
En cuanto al tiempo, el que predomina es el presente (91\%), seguido por el futuro perifrástico (3\%). La distribución temporal de todas las formas se puede observar en la Figura 3. En la Tabla V se presentan algunos ejemplos.

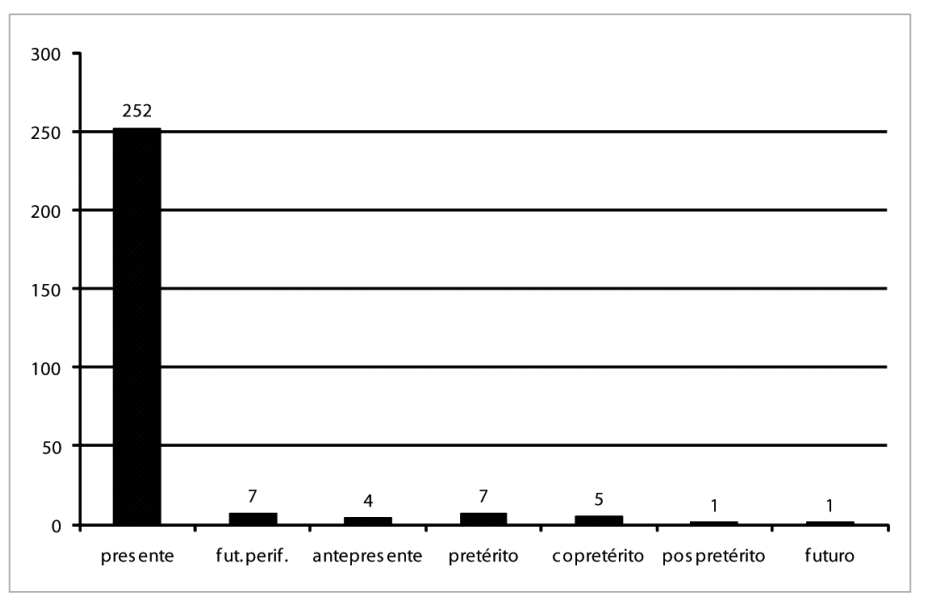

Figura 3. Tiempo de las formas evidenciales de $2^{\mathrm{a}}$ persona singular.

Tabla V. Ejemplos de segunda persona singular con interpretación no deíctica según tiempo.

\begin{tabular}{|l|c|}
\hline \multicolumn{1}{|c|}{ Ejemplos } & Tiempo \\
\hline $\begin{array}{l}\text { creo que nadie de aqui ha hecho el servicio o ha estado en la milicia, y si no } \\
\text { ESTAS, entonces no sabes... }\end{array}$ & presente \\
\hline $\begin{array}{l}\text { tienen que admitir q con esa wea, de ahora en adelante vos caminai y } \\
\text { TE VAI A TROPEZAR con un wn curao. Pero la wea rara es que ahora } \\
\text { dejan tomar en la calle pero restrigen los horarios de ventas de alcohol. son } \\
\text { consecuentes al hacer eso?=?? }\end{array}$ & $\begin{array}{c}\text { Futuro } \\
\text { perifrástico }\end{array}$ \\
\hline $\begin{array}{l}\text { Se deberia pagar la pena con trabajo social, y suspencion de documento de } \\
\text { por vida si es reincidente,pero eso de dormir en una carsel en la noche,no } \\
\text { le veo el veneficio,te creo pesfectamente si HAS TENIDO un choque,as } \\
\text { atropellado a alguen,etc,en esos casos si. }\end{array}$ & Antepresente \\
\hline $\begin{array}{l}\text { Cuando te despiden, sientes que FALLASTE, que no estás a la "altura", } \\
\text { lo que genera un terrible estado de inseguridad que te impide afrontar de } \\
\text { buena manera la búsqueda de otras alternativas laborales. }\end{array}$ & Pretérito \\
\hline $\begin{array}{l}\text { hay unos servicios que arriendas una casilla en gringolandia y estos tipos TE } \\
\text { TRAIAN tu encargo desde alla, no era tan caro, estaba en la SOFTEL, pero } \\
\text { no me acuerdo el nombre.. }\end{array}$ & Copretérito \\
\hline $\begin{array}{l}\text { El de diego torres si te autosugestionas PODRIAS ESCUCHAR algo, pero } \\
\text { = suena muy falso. }\end{array}$ & Pospretérito \\
\hline $\begin{array}{l}\text { Se trata de algo sobre lo cual tienes noticia de que existe sólo porque te } \\
\text { lo han dicho y que sabes, dada s las características de lo dicho, que nunca } \\
\text { PODRÁS ABARCAR eso de lo que sabes }\end{array}$ & Futuro \\
\hline
\end{tabular}


De las formas de $2^{\text {a }}$ persona singular con interpretación evidencial, la mayor parte posee aspecto imperfectivo (96\%). No obstante, se encuentran también casos correspondientes al aspecto perfectivo $(3 \%)$ y al perfecto $(1 \%)$.

Existen, también, otros elementos que confluyen para lograr una interpretación evidencial: los llamados "activadores genéricos", que pueden ser verbos auxiliares como "deber" o "tener", y algunas construcciones adverbiales y condicionales. En el corpus se rastreó la presencia de estos activadores y se obtuvieron los siguientes resultados:

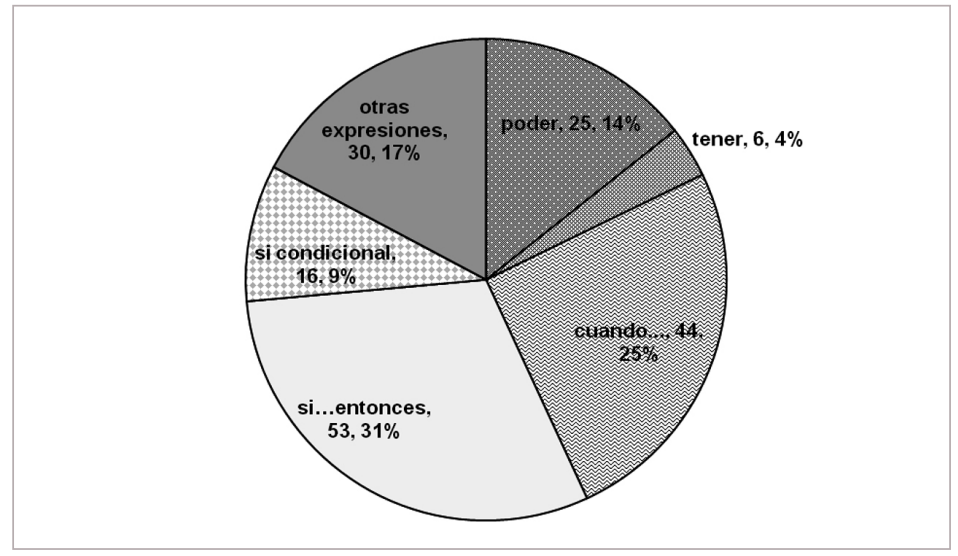

Figura 4. Activadores genéricos presentes en los contextos de segunda persona singular interpretados como evidenciales.

Otros activadores interesantes son los adyacentes del tipo "en este lugar" o "en esta época", que corresponden a un 16,9\% de los casos. Como ejemplos de esto último se entrega el siguiente enunciado:

(29) Chile; donde tu le dices a un Juez de la Corte Suprema que esta equivocado y te manda preso sin derecho a pataleo.

Respecto a la capacidad de las formas de $2^{\text {a }}$ persona singular para coaperecer con otras formas indefinidas, se tiene que en un alto porcentaje de los casos (90\%) ésta no se manifestaba. Los casos de coaparición con mayor ocurrencia corresponden a los de "se" (6\%) y a los de "uno" (3\%). Un ejemplo interesante en el que se puede apreciar la coaparición se entrega en (30):

(30) Me gusta el desafío de la competencia, eso te hace trabajar mejor. Si uno trabaja compitiendo con gente que no es buena en lo que hace, se queda dormido. Uno quiere comparar su trabajo con los mejores, ser parte de ese grupo. 
Un aspecto que llama la atención es la presencia, en el contexto inmediato de las formas de $2^{\text {a }}$ persona singular con interpretación evidencial, de formas de primera persona singular y plural. En 122 casos del corpus (44\%), la primera persona resultó relevante en la interpretación en cuestión. Esto hace pensar que podría ser un factor importante para comprender el significado de la unidad que se está investigando.

\section{INTERPRETACIÓN DE RESULTADOS}

Una de las primeras interpretaciones que se pueden establecer, a partir del análisis de las formas de $2^{\text {a }}$ persona singular con interpretación evidencial, se refiere al tipo de significado que transmiten las formas analizadas. Para entenderlo se puede partir de los ejemplos (31) y (32):

(31) Muchas veces la vida nos da sorpresas.. Muchas veces terminas trabajando en lo que no imaginabas.

En (31) el hablante utiliza la forma «nos» que corresponde a un caso de un clítico de $1^{\text {a }}$ persona plural en función de objeto indirecto y luego varía a una $2^{\text {a }}$ persona singular con la que se refiere no a un hablante, sino a todos aquellos que involucraba el primer uso del clítico de $1^{\text {a }}$ persona plural. Con esto se tiene que el acceso a la información es compartido y que la experiencia, que era personal ( $1^{\mathrm{a}}$ persona plural) se presenta como si no lo fuera, al proyectarla hacia otros que pudieran encontrarse en la misma situación. Para comprender mejor esto se entrega otro ejemplo tomado del mismo corpus:

(32) Nunca perdimos la fe. Hambre-hambre no pasamos, pero apreturas sí, y hartas. Por Dios que enriquece la pobreza, te enseña a apreciar lo que a ti te ha tocado. Vivimos dificultades pero siempre fui feliz.

En el ejemplo anterior se observan varios verbos en primera persona plural "perdimos», "pasamos», "vivimos», lo que hace pensar que el hablante efectivamente participó en la experiencia relatada. Estas formas indican que la fuente de la información es personal y que el acceso a ella es privativo de quienes vivieron esa experiencia. Sin embargo, cuando el hablante escoge luego una forma de $2^{\text {a }}$ persona singular no deíctica (en el ejemplo citado, los pronombres «te»), presenta el evento como posible de ser compartido con otros que no han tenido acceso a esa experiencia, es decir el acceso a la información se vuelve compartido y la experiencia se presenta desligada de lo personal.

Si se realiza una comparación con el pronombre «uno», que ya había sido es- 
tudiado en el marco de la evidencialidad, situando su significado en el eje acceso a la información como universal y la fuente de información como personal, se observa que las formas de $2^{a}$ persona singular se diferenciarían de él en que las primeras en el continuo de la fuente de la información son menos personales (se proyectan hacia otros) y en que en acceso a la información son menos universales (compartidas).

Esta interpretación tiene relación con la propuesta de Hollaender Jensen (2005) acerca de las diferencias entre «tú» y «yo». Él señala que respecto de «uno», «tú» es más empático, en tanto apela al interlocutor a que participe de la experiencia relatada, y también se liga de manera mucho menos directa que "uno" con la primera persona.

Lo anterior es apoyado por Vila (1987) con la idea de la transmisión de experiencias a otros, que en opinión de Fernández Ramírez (1986) sería desde una persona concreta, elegida como ad exemplum hacia una predicación de validez universal. Con el «uno» se parte de forma inversa: el hablante comienza con un supuesto dado ya como universal, en el que pueden instalarse cómodamente el hablante y el receptor.

De esta forma, el significado de esta unidad puede explicarse como una estrategia en la que una hablante utiliza una forma de $2^{a}$ persona singular para proyectar a otros una experiencia personal, como si no lo fuera, con la intención de persuadir empáticamente a otros que podrían encontrarse en una situación similar a la suya. Así, en el eje fuente de la información, ésta se presenta como desligada de lo personal y el acceso a la información se plantea como compartido. El significado anteriormente explicado queda graficado en el siguiente esquema:

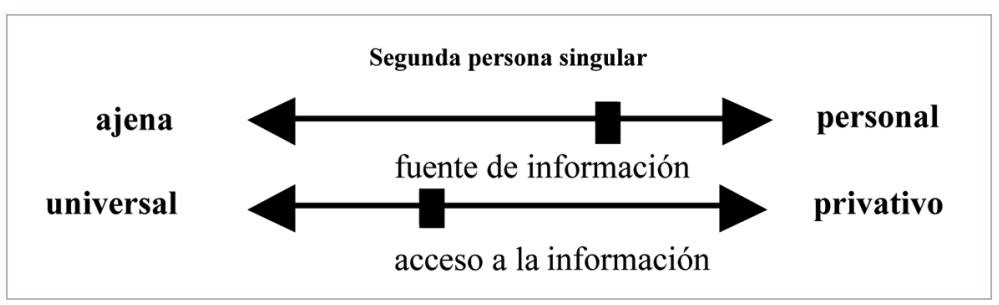

Figura 5. Significado evidencial que transmite la forma de $2^{\text {a }}$ persona singular en los ejes fuente de información y acceso a la información.

Respecto de los factores sintácticos y morfológicos que pueden influir en la interpretación evidencial se llega a interpretaciones bastante interesantes, sobre todo en relación al tiempo, al aspecto y a los activadores genéricos.

Como se explicó anteriormente, Hernanz (1990) propone que los singulares 
arbitrarios - los que hemos circunscrito al dominio de la evidencialidad- serían defectivos, ya que al poseer un valor genérico no son compatibles con entornos temporales y aspectuales de carácter puntual, como por ejemplo el pretérito. Sin embargo, en el corpus analizado se encontraron unidades de tiempo pretérito que, igualmente, permiten una interpretación evidencial en casos como (33) y (34):

(33) Sin olvidar que ser despedido en este país es un estigma, sin dudarlo todo tu entorno piensa que fué por mal profesional, por un "condoro" o que estuviste metido en algo turbio.

(34) En esta sociedad adoradora de la belleza, aunque no tengas ni cuarto medio, si tienes una "carita agraciada", te salvaste de lavar wateres, cabrita...

Hernanz (1990) no realiza una distinción clara entre tiempo y aspecto léxico y gramatical, lo que puede generar confusiones teóricas ${ }^{4}$. La hipótesis de Hernanz sobre la invalidación de la interpretación evidencial es aplicable sólo al aspecto no puntual, pero no al tiempo pretérito. La influencia que ejercen los "activadores genéricos" y el aspecto no puntual del contexto de las unidades de segunda persona singular es mucho mayor que la del tiempo en que se presente la forma para ser interpretada como evidencial.

En los casos (33) y (34) hay elementos como "en esta sociedad", "en este país" que permiten que la experiencia relatada por el hablante sea susceptible de ser compartida por otros que se encuentren en la misma situación (quienes vivan en una sociedad adoradora de la belleza o que trabajen en Chile). Al mismo tiempo, en (34) se encuentra la construcción "si... entonces", que también resulta relevante en la interpretación evidencial (si tienes una cara bonita, entonces te salvaste...).

Otro aspecto interesante es la influencia de "frases hechas", "refranes" o "construcciones fijas" en los contextos de aparición de las formas evidenciales, ya que entregan una idea de "genericidad", en tanto la información sería compartida por todos. Esto hace más factible la extensión de una experiencia personal hacia otros. Lo anterior se ejemplifica en (35):

(35) el trailer en el cine causó risa e incredulidad $\wedge^{\wedge} \mathrm{O}^{\wedge}$ mi hermano chico te sale con un mejor argumento.

${ }^{4}$ Como bien señala uno de los evaluadores anónimos de este artículo, en el caso de "estuviste metido", el aktionsart del predicado es estativo, lo que por definición excluiría su interpretación puntual; sin embargo, me parece pertinente destacar que el aspecto morfológico del verbo sí privilegia esta lectura. Obsérvese que el hablante podría haber escogido en su lugar conjugar el verbo en pretérito imperfecto: "estabas metido en algo turbio", en que el aktionsart de estado sería coherente con el aspecto imperfectivo del verbo, pero decide no hacerlo, lo que contraría la predicción de Hernanz. 
En (35) hay una frase hecha "salir con algo" en "mi hermano chico te sale con algo mejor" que se usa para calificar un elemento como poco valioso o poco deseable (generalmente éste se vincula de alguna manera con una actividad cognitiva). Así, claramente, el "te" presente en el ejemplo no hace referencia a un oyente, sino a "cualquiera", "a todos".

La coaparición con otras unidades con significado genérico también se encontró en el corpus, en casos como (36):

(36) te da mas energia y no te cansay tanto, aunq tambien estan las bebidas isotonicas que hacen que uno se recupere más rapido ;)

En ejemplos como (36) la presencia del pronombre "uno" es la que permite interpretar los casos de $2^{\text {a }}$ persona como evidenciales. Nótese el contraste en la interpretación de las formas de $2^{\text {a }}$ persona singular, sin la presencia del pronombre "uno":

(37) te da mas energia y no te cansay tanto, aunq tambien estan las bebidas isotonicas que hacen que te recuperes más rapido ;)

En un caso como (37) la interpretación evidencial no es tan claramente posible, lo que confirma la gran influencia que tiene la coaparición para la significación compartida. La presencia del pronombre «uno» es lo que liga al enunciado con el hablante, denota que existe una experiencia de la que él es parte (el conocimiento de las bebidas isotónicas), la que es proyectada hacia otros.

En el corpus se encontró un caso interesante que no se pudo interpretar fácilmente como evidencial o deíctico, es el presentado en (38):

(38) La concertación le debe mucho a Don Patricio, durante estos 12 años se han construido grandes obras que tu las puedes ver donde vallas, pero, algunos le echan una mosca a la torta y arman un escandalo por la mosca. Miremos la gran obra que se ha realizado, hay una valla muy alta para el próximo presidente, quienes hablan del cambio dicen que la mosca de la torta la taparán con crema. Gracias Don Patricio, por su entrega, valor y espíritu de servicio.

En (38) se observan tres elementos de $2^{a}$ persona singular, el pronombre "tú" en función sujeto y las formas verbales "puedes" y "vayas". Luego se presenta una forma en primera persona plural "miremos", que podría influir en la interpretación de las unidades de $2^{a}$ persona singular como evidenciales en tanto que haría pensar que el "tú" no se refiere a un oyente, sino a todos aquellos involucrados en el tema que se está discutiendo.

Podría pensarse también que la interpretación evidencial se ve disminuida por 
la presencia del pronombre personal explícito. Sin embargo, se cree que esta unidad en función sujeto no influye, lo que se demuestra en un caso como (39), que igualmente es interpretada como evidencial:

(39) Tenemos el derecho a preguntar y si vemos que no hay una sinceridad, que el maestro no es consecuente con las enseñanzas o que tiene un sistema de vida contrario a lo que está diciendo, tu cambias de maestro o absorbes las partes que te sirven.

En (39) se observa que al igual que en (38) hay un pronombre "tú" en función sujeto y que también existe una vinculación del enunciado con el hablante a través de una primera persona plural (tenemos). Sin embargo, en (39) hay otros elementos que confluyen con la primera persona plural y generan una interpretación evidencial de la unidad de $2^{\text {a }}$ persona singular, como la estructura "si... entonces". (38) no posee una interpretación claramente evidencial, pero no debido a la presencia del pronombre "tú", sino porque los elementos activadores de la genericidad están ausentes, lo que reafirma la idea de la importancia de los activadores genéricos. (38) muestra la influencia que tiene el contexto en la interpretación evidencial de las formas de segunda persona singular, además de recordar que el fenómeno de la evidencialidad puede ser considerado una cuestión de grado. De ahí que la propuesta de los ejes continuos sea preferible frente a clasificaciones rígidas.

Se cree, entonces, que el pronombre "tú" no invalida la interpretación evidencial y que tampoco habría matices de significado distintos generados por su presencia o ausencia en una forma interpretada como evidencial.

Si bien la mayor parte de las formas de $2^{\text {a }}$ persona singular poseen una función sintáctica verbal (56\%), se cree que ésta no influye en la interpretación de ésas como evidenciales.

\section{CONCLUSIONES}

A partir de la interpretación de los resultados del apartado anterior se puede concluir que la $2^{\text {a }}$ persona singular posee una interpretación evidencial que se ve favorecida por aspectos sintácticos y morfológicos.

Los entornos aspectuales y temporales de los casos interpretados como evidenciales poseen, la mayoría, la característica de ser no puntuales (tiempo presente y aspecto imperfectivo). Sin embargo se detectaron formas con interpretación evidencial que se encontraban en entornos puntuales (pretérito) y cuyos aspectos eran perfectivos. Con esto se concluye que los entornos temporales y aspectuales no puntuales favorecen la interpretación evidencial de las formas de $2^{a}$ persona singular, pero no son un requisito para que ésta pueda efectuarse. 
Se llegó a la conclusión de que el factor más influyente en la interpretación evidencial es la presencia de activadores genéricos que permiten que una experiencia personal pueda ser generalizada a otros. Los activadores más frecuentes fueron las estructuras condicionales del tipo "si... entonces" y adyacentes circunstanciales como "en este país".

La presencia del pronombre personal de $2^{a}$ persona en función sujeto se registró en muy pocas oportunidades (17 casos), lo que se relaciona con la propiedad del español de ser una lengua que no presenta obligatoriamente un sujeto expreso o de ser una lengua de sujeto nulo. La presencia del pronombre no resultó relevante en ninguno de los casos evidenciales y tampoco se observaron matices de significado distinto generados por su ausencia o presencia.

La coaparición de las formas de $2^{\text {a }}$ persona singular con otras formas indefinidas es posible y frecuente en el caso en cuestión. Se cree que la coaparición favorece la interpretación evidencial, al permitir, igual que los activadores genéricos, la generalización de una experiencia personal. Llama la atención también la presencia de enunciados vinculados con la primera persona en los contextos de las formas de $2^{a}$ persona singular. Esto posibilitaría relacionar al hablante con la experiencia que éste quiere extender a otros.

Se piensa que la función sintáctica que desempeñe la forma de $2^{a}$ persona singular no resulta relevante para la interpretación evidencial.

Con todo lo anterior se demuestra que la interpretación evidencial de la $2^{\text {a }}$ persona singular obedece a una constelación de factores tales como la presencia de activadores genéricos, el tiempo, el aspecto, la coaparición con otras formas genéricas y la relación con la primera persona que haga que la información, siendo personal, sea susceptible de ser proyectada hacia otros que se encuentren en la misma situación que el hablante. Se puede concluir que las formas analizadas sí transmiten un significado evidencial que es relevante en los ejes fuente de la información y acceso a la información y que sí existen elementos sintácticos y morfológicos sistemáticos que influyen en la interpretación evidencial, lo que justifica el estudio de esta forma en el marco de la evidencialidad.

\section{REFERENCIAS}

Aikhenvald, Alexandra. 2004. Evidentiality. Oxford: Oxford University Press.

Barrajón, Elisa. 2005. "Un caso de impersonalidad semántica: el uso de los llamados 'singulares arbitrarios' en corpora orales", en Estudios de lingüistica 19, pp.47-64.

Benveniste, Emile. 1997 [1971]. "La naturaleza de los pronombres" y "Estructura de las relaciones de persona en el verbo". En Problemas de lingüistica general I, decimonovena edición en español. México: Siglo XXI, pp. 161-178. 
Bermúdez, Fernando. 2005. Evidencialidad: la codificación lingüística del punto de vista. Tesis doctoral. Estocolmo, Suecia: Universidad de Estocolmo.

Boas, Franz. 1938. "Lenguaje”. En F. Boas (ed.). General Anthropology. Boston: D. C. Heath \& Company, pp. 124-145.

Briz, Antonio. 1998. El español coloquial: situación y uso. Segunda edición. Madrid: Arco Libros.

Bybee, Joan. 1985. Morphology: a study of the relationship between meaning and form. Amsterdam: John Benjamins.

Fernández Ramírez, Salvador. 1986. "Las personas gramaticales. Comprehensión y transposición. Las personas generales", en Gramática española. El verbo y la oración. Volumen 4. Madrid: Arco Libros, pp. 21-57.

Fernández Soriano, Olga. 1999. "El pronombre personal. Formas y distribuciones. Pronombres átonos y tónicos". En I. Bosque \& V. Demonte Gramática descriptiva de la lengua española (GDLE). Madrid: Espasa, pp. 1209-1273.

González, Carlos y Lima, Pablo. 2009. "Estrategias de expresión de la evidencialidad en la argumentación oral en la sala de clases", en Revista Signos, estudios de lingüistica 71, 42, pp. 295-315.

Hernanz, María Luisa. 1990. "En torno a los sujetos arbitrarios: la 2a persona del singular”, en V. Demonte y B. Garza Cuarón (eds.). Estudios de lingüistica de España y México. México: UNAM, pp. 151-178.

Hollaender Jensen, Mikkel. 2002. "La referencia en algunas expresiones impersonales. Diferentes lecturas de uno y la segunda persona del singular", en Romansk Forum 16, 2, pp. 127-138.

Santo Thomas, Domingo de. 1951 [1560] Grammatica o Arte de la lengua general de los indios de los reynos del Perú. Lima: Edición del Instituto de Historia.

Vila, María Rosa. 1987. "La segunda persona gramatical en función no deíctica", en Revista Española de Lingüistica 17 (I), pp. 57-68. 